\title{
Modeling Methane Adsorption in Interpenetrating Porous Polymer Networks
}

\author{
Richard L. Martin, ${ }^{\dagger}$ Mahdi Niknam Shahrak, ${ }^{\ddagger}$ Joseph A. Swisher, $^{\S}$ Cory M. Simon, ${ }^{\S}$ Julian P. Sculley, " \\ Hong-Cai Zhou," Berend Smit, ${ }^{\S}$ and Maciej Haranczyk ${ }^{\dagger}{ }^{\dagger}$ \\ ${ }^{\dagger}$ Computational Research Division, Lawrence Berkeley National Laboratory, Berkeley, California 94720, United States \\ ${ }^{*}$ Department of Chemical Engineering, Quchan Institute of Engineering and Technology, P.O. Box 84686-94717, Quchan, Islamic \\ Republic of Iran \\ ${ }^{\S}$ Department of Chemical and Biomolecular Engineering, University of California at Berkeley, Berkeley, California 94720, United \\ States \\ "Department of Chemistry, Texas A\&M University, College Station, Texas 77842, United States
}

\section{Supporting Information}

ABSTRACT: Porous polymer networks (PPNs) are a class of porous materials of particular interest in a variety of energy-related applications because of their stability, high surface areas, and gas uptake capacities. Computationally derived structures for five recently synthesized PPN frameworks, PPN-2, -3, -4, -5, and -6, were generated for various topologies, optimized using semiempirical electronic structure methods, and evaluated using classical grand-canonical Monte Carlo simulations. We show that a key factor in modeling the methane uptake performance of these materials is whether, and how, these material frameworks interpenetrate and demonstrate a computational approach for predicting the presence, degree, and nature of interpenetration in PPNs that enables the reproduction of experimental adsorption data.

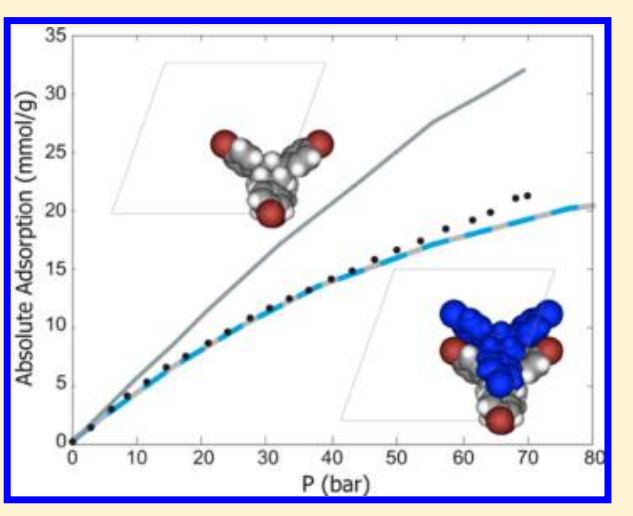

\section{INTRODUCTION}

Gas storage and separation is of increasing importance to the development of new and cleaner energy technologies, with the storage of natural gas for motor vehicles and carbon dioxide capture $^{1-3}$ being flagship examples. Selective and efficient adsorbents based on porous media represent one class of possible technology-enabling materials. While several classes of adsorbents have established applications in large-scale processes (e.g., activated carbon or zeolites), recent efforts in chemical synthesis have produced a large range of novel materials, such as porous polymer networks (PPNs) (or porous aromatic frameworks, PAFs ${ }^{4,5}$ ) and metal-organic frameworks (MOFs). One key aspect of these new classes of materials is that their structures can be tailored for specific applications. ${ }^{6}$ With the rapid growth of available computing power, the process of simulating hypothetical structures and using these simulations to predict experimental isotherms has taken the lead in aiding both the rational design of new materials and better characterization of existing materials. ${ }^{7}$ One class of materials that has been more difficult to characterize because it forms an amorphous powder, PPNs, has shown potential for carbon dioxide capture $^{8,9}$ and high-pressure gas storage. ${ }^{10,11}$ PPNs are of particular interest because they combine the advantages of inexpensive and stable polymers with those of ultrahigh-surfacearea MOFs and can thus be targeted for vehicular methane storage applications. ${ }^{12}$
Although computer simulations can be used to predict isotherms with an accuracy similar to that of experiment, computer-aided rational design of new materials and better characterization of existing materials ${ }^{7}$ depend largely on generating models with the correct geometrical and physical parameters. This task is not straightforward in the case of PPN/ PAF materials, because such materials do not have well-defined crystal structures. However, it is important to note that PPNs are very different from amorphous polymers, because shortrange order can be expected in these materials, as has been demonstrated for PAF-1. Short-range order in PAF-1 can be approximated with a crystalline model, which has been shown to enable the accurate simulation of nitrogen adsorption. ${ }^{4}$ However, an amorphous model can also enable close reproduction of experimental measurements. ${ }^{13}$ In this work, we aim to show that, although multiple distinct models for an individual porous polymer can produce similar data, the explicit consideration of interpenetrated network structures (i.e., one or more nested networks) is necessary for PPNs that exhibit large pores.

Framework interpenetration ${ }^{14}$ (interweaving or catenation ${ }^{15}$ ) is typically considered as a negative agent in crystal engineer-

Received: July 12, 2013

Revised: $\quad$ August 28, 2013

Published: September 3, 2013 
ing ${ }^{16,17}$ because it results in a reduction of accessible pore volume and an increase in framework density. However, from a gas storage and separation point of view, these are two opposing factors that can be controlled to obtain materials with the optimal performance; increasing the framework density can create more adsorption sites but can also decrease the number of molecules that can be adsorbed. ${ }^{16,17}$ Therefore, it is important to control the level of interpenetration and, thereby, the porosity of a material, as it can potentially improve performance. For instance, Yang et al. synthesized a partially interpenetrated MOF exhibiting the unusual property of increased selectivity for carbon dioxide. ${ }^{18}$ An important design target for vehicular methane storage applications is achieving a balance between the competing objectives of gravimetric and volumetric storage, to achieve an optimal material. Variation of temperature and concentration during synthesis and utilization of liquid-phase epitaxy on an organic template are some of the common procedures employed to control interpenetration. ${ }^{16,17}$ At present, there is very little quantitative understanding of whether a given material will interpenetrate. Herein, we describe a model that can be used to predict the degree and exact mode of interpenetration in PPNs and validate our predictions against experimental data. Our model, through explicit consideration of the effects of interpenetration, can lead to more efficient design of next-generation porous polymer materials.

\section{METHODS}

The five PPNs examined in this work were synthesized through the self-assembly of tetrahedral substrates (Figure 1). Following

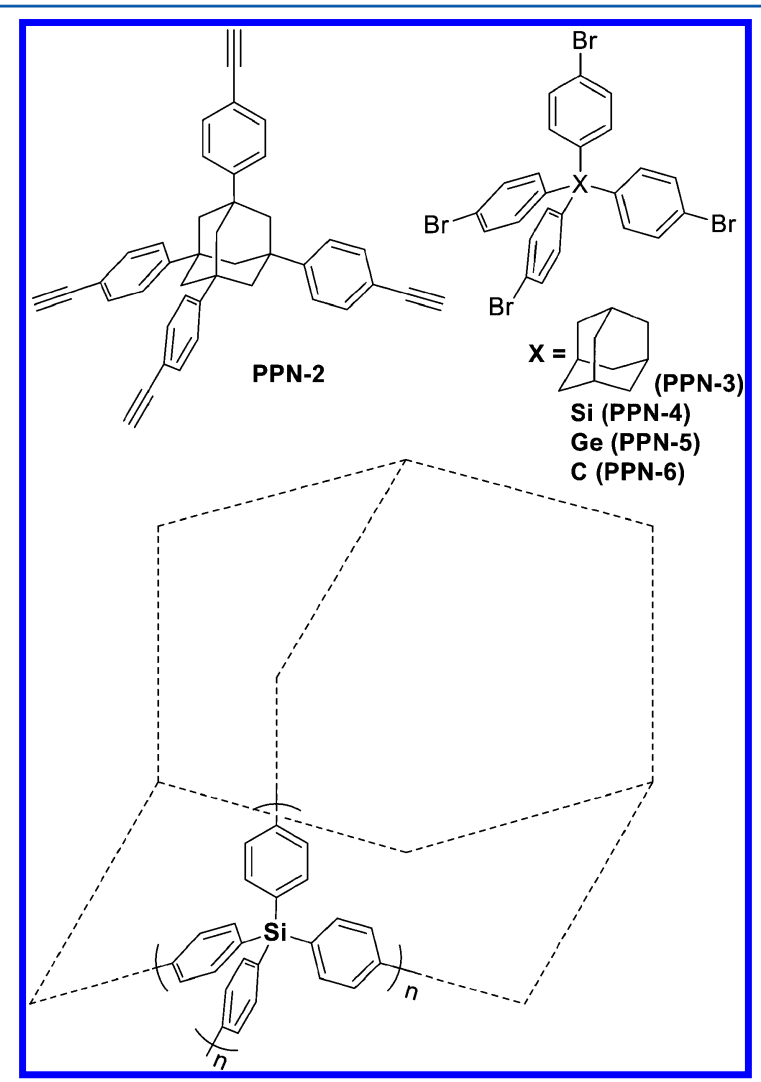

Figure 1. Top: Monomers used to synthesize PPN-2-PPN-6. Bottom: Schematic connectivity for dia net depicted using PPN-4 monomer. the work of Ben et al., ${ }^{4}$ we assume short-range order in these structures. As a result, the materials are modeled by small ordered fragments with periodic boundary conditions, making the models equivalent to idealized crystal structures. Porous frameworks made up of tetrahedral building units can exhibit a variety of net topologies; ${ }^{19}$ of these, the diamond net $\left(\mathrm{RCSR}^{20}\right.$ symbol dia) exhibits the highest symmetry (transitivity 1111), and accordingly, it has been demonstrated to be, by far, the most prolific. ${ }^{21,22}$ Therefore, we model PPN materials using the dia topology; ${ }^{10}$ however, we also consider alternative, lowersymmetry topologies arising from tetrahedral building units, namely, sod (1121) and lon (1222), with results for these alternative nets provided in the Supporting Information. The structure models of each PPN were created by repeating the unit of the monomer molecule according to the underlying topology, and in the cases of PPN-3-PPN-6, removing the bromo substituents to accurately represent the reaction conditions (Figure 1).

At present, experimental methane adsorption data exist only for PPN-2, -3, and -4. Despite the significant similarity among these materials, adsorption experiments indicate significantly lower surface area and uptake in PPN-3 compared to PPN-4, an effect further exaggerated in PPN-2 and speculated to be due to interpenetration of PPN-3 and PPN-2. ${ }^{10}$ Indeed, whereas noninterpenetrated models, either crystalline ${ }^{4}$ or amorphous, ${ }^{13}$ can reproduce the experimental data for PPN-4, we found in this work that noninterpenetrated structure models for PPN-3 cannot reproduce the experimental isotherms (see Results and Supporting Information for sod and lon nets). Hence, we explore the hypothesis that certain PPNs interpenetrate and, in particular, that the specific nature or mode of framework interpenetration is important in reconciling experimental methane adsorption behavior with that achieved from simulation.

Crystalline materials exhibiting the dia net are known to produce equally spaced nets upon interpenetration (i.e., maximal crystallographic symmetry, dia-c net). ${ }^{23}$ However, in the case of PPN-3, we found that assuming this model does not improve the fit to the experimental behavior and, indeed, results in starkly different performance (Figure $2 \mathrm{~b}$ ). Specifically, the equally spaced nets in the dia-c model result in overlapping potentials and binding interactions and, hence, noticeably higher methane uptake at low pressure with respect to the dia net, a phenomenon that clearly is absent from the experimental results. Hence, we conclude that the structure of PPN-3 must exhibit a lower pore volume than the dia net to match the saturation loading from experiment, but must not exhibit greater solid-methane interactions ( $\mathrm{vdw}$ potential overlap), as this would enhance the uptake at the lower pressures. Therefore, a systematic computational search of possible modes of interpenetration of dia net models was conducted to evaluate alternative interpenetrated structures. We note that the sod and lon nets are mathematically precluded from selfinterpenetration because they are not self-dual, and they were therefore omitted from this search.

An exhaustive search of possible interpenetrated dia networks for each PPN was conducted. Interpenetrated frameworks were generated computationally by duplicating the underlying net and positioning it with a particular geometric shift relative to the original; each permissible shift (i.e., shifts resulting in atomic collisions between nets were rejected as described in the Supporting Information) in the Cartesian axes to a $1-\AA ̊$ resolution was performed independ- 

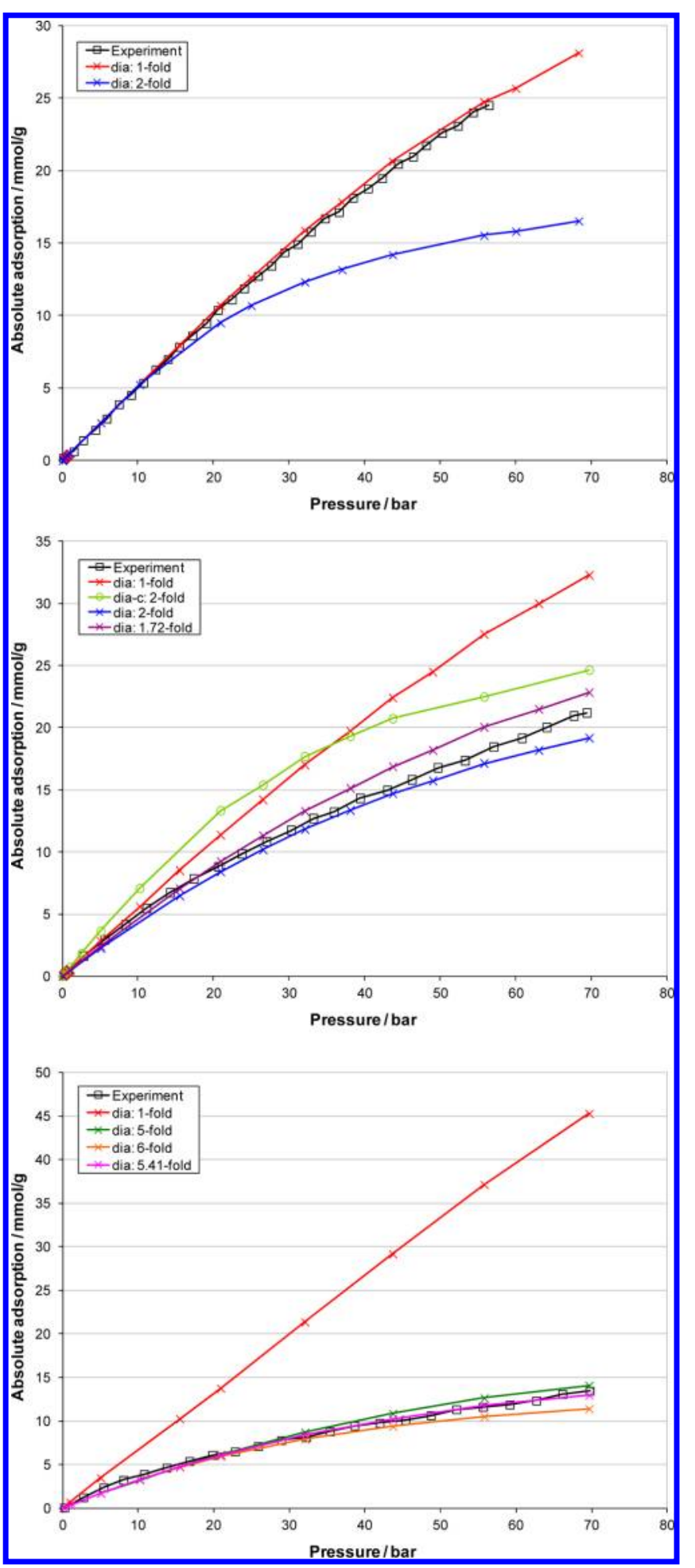

Figure 2. Methane adsorption isotherms at $295 \mathrm{~K}$ for PPN-4 (top), PPN-3 (center), and PPN-2 (bottom).

ently, taking into account the periodic boundary conditions. Each interpenetrated structure was optimized by the semiempirical PM6-DH2 method. $^{24,25}$ (We note that, to permit full, independent relaxation of the atomic positions in each net, all structures were modeled with the P1 space group.) For each interpenetration model, the resulting framework stability was analyzed; the most stable interpenetrated framework was considered to be the most probable and was selected.
We observed that, for each PPN, the selected, lowest-energy structure represents a close geometrical arrangement of networks, wherein the van der Waals interactions between each network are maximized. We note that this arrangement of nets is in stark contrast to the dia-c model of equally spaced nets observed for crystalline interpenetrating materials. ${ }^{23}$ All interpenetrated structures considered in the following discussion were fully optimized using such a geometry as the starting point; crystallographic atom coordinate data in .cif format are provided in the Supporting Information.

Geometric analysis of each PPN was conducted using the Zeo++ software suite. ${ }^{26}$ The selected, lowest-energy interpenetrated framework arrangement was also observed to exhibit the lowest probe-accessible surface area. Accordingly, identification of low-surface-area network arrangements is postulated as an efficient computational method for identifying energetically favorable interpenetrated states in PPN materials.

It has been observed that, in crystalline materials of the pcu net, there is a direct relationship between net edge length (i.e., pore size) and propensity for interpenetration. ${ }^{27}$ A similar argument is proposed here. The restricting pore diameters of each material were calculated assuming the van der Waals radii of each atom and rigid structures (Table 1). PPN-3, by

Table 1. Restricting Pore Diameters of the dia Net PPNs Studied in This Work

\begin{tabular}{ccccccc} 
& & \multicolumn{5}{c}{$n$-fold interpenetrated structures } \\
\cline { 3 - 7 } PPN- & basic net & 2 & 3 & 4 & 5 & 6 \\
2 & 24.43 & 20.89 & 17.10 & 12.89 & 9.54 & 9.44 \\
3 & 15.49 & 12.14 & & & & \\
4 & 11.40 & & & & \\
5 & 11.40 & & & & \\
6 & 9.47 & & & & \\
\hline
\end{tabular}

comparison to PPN-4-PPN-6, exhibits a significantly larger restricting pore diameter, whereas its monomer, comprising an adamantane core, is not significantly larger. Hence, the larger pore diameter-to-monomer ratio in PPN-3 is postulated to indicate greater ease and likelihood of interpenetration in PPN3 compared to PPN-4-PPN-6. This effect is further exaggerated in PPN-2 because its restricting pore diameter is over $9 \AA$ greater than that of PPN-3, whereas its monomer is only slightly larger (Figure 1). Hence, multiple levels of interpenetration might occur in PPN-2. PPN-2 structures with increasing levels of interpenetration were generated following the above procedure (Table 1), and it was observed that the restricting pore diameter for a 4-fold interpenetrated PPN-2 is still larger than that of interpenetrated PPN-3. We therefore postulate that at least 5-fold interpenetration for PPN-2 is achievable.

Methane adsorption for each PPN was analyzed by grandcanonical Monte Carlo simulations (details are provided in the Supporting Information). Simulation results are illustrated in Figure 2; as can be observed, noninterpenetrated structures, exhibiting larger pore volumes, consistently exhibit higher capacities to adsorb methane than corresponding interpenetrated structures, which is consistent with the literature. ${ }^{28}$ Table 2 summarizes the structural properties of each PPN. Pore volumes were obtained from nitrogen adsorption isotherms at $77 \mathrm{~K}$, from either experiment or simulation. 
Table 2. Structural Properties of the dia Net PPNs Studied in This Work ${ }^{a}$

$\begin{array}{lccc}\text { PPN }^{b} & \begin{array}{c}\text { framework density } \\ \left(\mathrm{g} / \mathrm{cm}^{3}\right)\end{array} & \begin{array}{c}\text { simulated pore } \\ \text { volume }\left(\mathrm{cm}^{3} / \mathrm{g}\right)\end{array} & \begin{array}{c}\text { experimental pore } \\ \text { volume }\left(\mathrm{cm}^{3} / \mathrm{g}\right)\end{array} \\ 2 & 0.089 & 10.24 & 1.26 \\ 2[2] & 0.178 & 4.83 & \\ 2[3] & 0.277 & 2.86 & \\ 2[4] & 0.371 & 1.96 & \\ 2[5] & 0.460 & 1.42 & \\ 2[6] & 0.553 & 1.04 & \\ 3 & 0.196 & 4.51 & \\ 3[2] & 0.392 & 1.94 & 3.04 \\ 4 & 0.284 & 2.88 & \\ 42] & 0.568 & 1.17 & 2.60 \\ 5 & 0.310 & 2.63 & \\ 5[2] & 0.620 & 1.07 & 3.05 \\ 6 & 0.324 & 2.56 & \\ 6[2] & 0.649 & 0.96 & \end{array}$

${ }^{a}$ All materials exhibit unit-cell angles of $\alpha=\beta=\gamma=90^{\circ} .{ }^{b}[n]$ indicates $n$-fold interpenetrated.

\section{RESULTS}

For PPN-4, the simulated methane adsorption isotherm of the noninterpenetrated structure compares favorably with the experimental data (Figure 2a). This suggests that the experimental structure is well-modeled by the noninterpenetrated dia net. Furthermore, it was observed that the pore volume of the noninterpenetrated dia structure differs by only $5 \%$ from experiment (Table 2). Indeed, our calculations show that the restricting pore diameter of the underlying PPN-4 net is too small for the constituent monomer to diffuse (Table 1; see Methods); accordingly, interpenetration is less likely in this material. We re-emphasize that dia is not the only possibility for PPN-4, because the sod and lon nets also produce good agreement with experiment (Supporting Information), as do amorphous models. ${ }^{13}$

Because the restricting pore diameters of PPN-5 and -6 are comparable to that of PPN-4 and the experimentally determined pore volumes are in good agreement with those calculated for noninterpenetrated structures, we conclude that the real structures of PPN-5 and -6 are likely to be noninterpenetrated. Hence, although no experimental methane adsorption data are available for these materials, we postulate that they exhibit methane adsorption behavior corresponding to the noninterpenetrated frameworks in the Supporting Information.

In contrast, the restricting pore diameter of PPN-3 is larger, and the simulated pore volume of the noninterpenetrated PPN3 structure model is much higher than the pore volume measured by experiment (Table 2), factors that suggest interpenetration. However, the interpenetrated structure exhibits a slightly lower pore volume than the experimental measurement. Considering that no residual bromine was detected in the synthesized materials, ${ }^{10}$ we do not consider the small discrepancy in pore volume to be caused by incomplete network formation (e.g., structural vacancies). Rather, we hypothesize that the real, noncrystalline structure is partially interpenetrated, ${ }^{18}$ comprising both interpenetrated and noninterpenetrated regions. By interpolating the $\mathrm{N}_{2}$ pore volumes reported in Table 2, the real PPN-3 structure is estimated to be approximately $72 \%$ interpenetrated. Comparison of methane adsorption data reinforces this hypothesis: The simulated noninterpenetrated structure substantially overpredicts the experimental methane adsorption isotherm, and the experimental behavior is seen to be closer to that of the interpenetrated state. Moreover, by taking a weighted average of simulated isotherms, 0.72 interpenetrated and 0.28 noninterpenetrated, we observed good agreement with experiment, particularly at lower pressures, leading us to conclude that this is the likely scenario (Figure $2 \mathrm{~b}$ ).

Finally, we found that the experimental pore volume of PPN2 lies between those of the 5- and 6-fold interpenetrated structures. Pore analysis indicated that at least 5-fold interpenetration was likely to be achievable (see Methods), and these observations were reinforced by agreement between the isotherms for the 5- and 6-fold interpenetrated structures and experiment (Figure 2c). As found for PPN-3, the best match for PPN-2 is a combination of two interpenetration levels; in this case, 5.41-fold interpenetration is suggested as the most likely structure.

\section{DISCUSSION}

It is important to recall that, in this work, we have modeled PPNs as (pseudo)crystalline materials. Experimental results for PAF-1 (PPN-6), in particular, powder X-ray diffraction, suggest little long-range order. The fact that we obtain such good agreement with the experimental data (e.g., PPN-4, and PPN3 ), using a force field that has been validated on other materials, illustrates that modeling these materials as (pseudo)crystalline is a reasonable approximation. Similarly, transmission electron microscopy images indicating a uniform pore diameter, infrared spectra indicating no $\mathrm{C}-\mathrm{Br}$ bonds (i.e., no incomplete polymerization), and fitting to the experimental density and $\mathrm{N}_{2}$ adsorption isotherm allowed Ben et al. to develop a crystalline model of PAF-1. ${ }^{4}$ However, as indicated by Trewin and Cooper, ${ }^{13}$ other noncrystalline structure models can be proposed with similar densities and low $\mathrm{C}-\mathrm{Br}$ bond contents. Clearly, further experimental data are necessary before a definitive statement can be made regarding the underlying structure of these materials, and we are hopeful that future experimental work can shed some light on this important question. Nevertheless, the fact that both the work of Ben et al. and the present work provide reasonable agreement gives us some confidence in our models.

In addition, our structure models are based on further assumptions such as that the structures exhibit no defects. In reality, this is not likely to be the case. For example, structures with partial interpenetration, as predicted for PPN-3, can be realized only as a system comprising an interpenetrated array of two defective (incomplete) nets.

Finally, we note that, in the absence of further experimental data for these materials, it is not possible to state definitively that all considered materials exhibit the dia net (transitivity 1111). For instance, lower-symmetry topological models based on tetrahedral vertices were also investigated (sod, transitivity 1121, and lon, transitivity 1222; see Supporting Information). However, despite similarities in methane uptake among the noninterpenetrated dia, sod, and lon models, we emphasize that sod and lon nets are not self-dual and are therefore mathematically precluded from self-interpenetration. Hence, it is not possible to reproduce the adsorption performance of either PPN-3 or PPN-2 using these alternative topologies, whereas the dia net models consistently enable reproduction of experimental data. 


\section{CONCLUSIONS}

We have presented a computational procedure by which the methane adsorption performance of PPN materials can be evaluated through (pseudo)crystalline structure models with appropriate consideration of low-symmetry interpenetration. We have shown that the likelihood of interpenetration in PPNs can be estimated based on restricting pore diameters; moreover, the structure of the interpenetrated state can be identified through a systematic computational analysis of the space of possible framework interpenetrations. Consistent with the hypothesis and conclusions from pore diameter and volume calculations, agreement between simulated and experimental isotherms confirms our claim regarding the real PPN-2, -3, and -4 structures. Furthermore, although noncrystalline, it has been shown that PPNs can be modeled successfully as crystal structures in grand-canonical Monte Carlo simulations. Very good agreement between simulation and experimental data was observed; it was shown that the likely structures of PPN-4, -5, and -6 are those of the noninterpenetrated form, whereas PPN3 is mostly of the interpenetrated form (0.72-fold) and PPN-2 is likely to exhibit multiple levels (5.41-fold) of interpenetration.

Our model is able to predict the maximum level of interpenetration. In practical terms, these results imply that the experimental isotherm will be bracketed by the isotherms predicted for this maximum level and one level lower. For many practical applications, such accuracy is sufficient to make a reasonable prediction of the performance of the material and can therefore lead to the design of improved materials for targeted applications.

\section{ASSOCIATED CONTENT}

\section{S Supporting Information}

Interpenetration methodology; simulation details; results for PPN-5, PPN-6, and alternative topologies; and combined crystallographic structure file for all materials. This material is available free of charge via the Internet at http://pubs.acs.org.

\section{AUTHOR INFORMATION}

\section{Corresponding Author}

*Fax: +1 510486 5812. Tel.: +1 510486 7749. E-mail: mharanczyk@lbl.gov.

\section{Author Contributions}

This manuscript was written through the contributions of all authors. All authors have given approval to the final version of the manuscript.

R.L.M. and M.N.S. contributed equally.

\section{Notes}

The authors declare no competing financial interest.

\section{ACKNOWLEDGMENTS}

R.L.M., M.H., and J.P.S were supported by the U.S. Department of Energy Advanced Research Projects AgencyEnergy (ARPA-E) under Award DE-AR0000249. H.-C. Z. were supported as part of the Center for Gas Separations Relevant to Clean Energy Technologies, an Energy Frontier Research Center funded by the U.S. Department of Energy, Office of Science, Office of Basic Energy Sciences, under Award DESC0001015. C.M.S. and B.S. are supported by the U.S. Department of Energy, Office of Basic Energy Sciences, Division of Chemical Sciences, Geosciences and Biosciences under Award DE-FG02-12ER16362. This research used resources of the National Energy Research Scientific Computing Center, which is supported by the Office of Science of the U.S. Department of Energy under Contract DE-AC02$05 \mathrm{CH} 11231$.

\section{REFERENCES}

(1) D’Alessandro, D. M.; Smit, B.; Long, J. R. Carbon Dioxide Capture: Prospects for New Materials. Angew. Chem., Int. Ed. 2010, 49 (35), 6058-6082.

(2) Li, J. R.; Sculley, J. P.; Zhou, H.-C. Metal-Organic Frameworks for Separations. Chem. Rev. 2012, 112 (2), 869-932.

(3) Smit, B.; Maesen, T. L. M. Molecular Simulations of Zeolites: Adsorption, Diffusion, and Shape Selectivity. Chem. Rev. 2008, 108 (10), 4125-4184.

(4) Ben, T.; Ren, H.; Ma, S. Q.; Cao, D. P.; Lan, J. H.; Jing, X. F.; Wang, W. C.; Xu, J.; Deng, F.; Simmons, J. M.; Qiu, S.; Zhu, G. Targeted Synthesis of a Porous Aromatic Framework with High Stability and Exceptionally High Surface Area. Angew. Chem., Int. Ed. 2009, 48 (50), 9457-9460.

(5) Schmidt, J.; Werner, M.; Thomas, A. Conjugated Microporous Polymer Networks via Yamamoto Polymerization. Macromolecules 2009, 42 (13), 4426-4429.

(6) Wriedt, M.; Sculley, J. P.; Yakovenko, A. A.; Ma, Y.; Halder, G. J.; Balbuena, P. B.; Zhou, H.-C. Low-Energy Selective Capture of Carbon Dioxide by a Pre-Designed Elastic Single-Molecule Trap. Angew. Chem., Int. Ed. 2012, 51 (39), 9804-9808.

(7) Lin, L.-C.; Berger, A. H.; Martin, R. L.; Kim, J.; Swisher, J. A.; Jariwala, K.; Rycroft, C. H.; Bhown, A. S.; Deem, M. W.; Haranczyk, M.; Smit, B. In Silico Screening of Carbon-Capture Materials. Nat. Mater. 2012, 11 (7), 633-641.

(8) Lu, W.; Sculley, J. P.; Yuan, D.; Krishna, R.; Wei, Z.; Zhou, H.-C. Polyamine-Tethered Porous Polymer Networks for Carbon Dioxide Capture from Flue Gas. Angew. Chem., Int. Ed. 2012, 51 (30), 74807484.

(9) Lu, W.; Yuan, D.; Sculley, J. P.; Zhao, D.; Krishna, R, Zhou, H.C. Sulfonate-Grafted Porous Polymer Networks for Preferential $\mathrm{CO}_{2}$ Adsorption at Low Pressure. J. Am. Chem. Soc. 2011, 133 (45), 18126-18129.

(10) Lu, W.; Yuan, D.; Zhao, D.; Schilling, C. I.; Plietzsch, O.; Muller, T.; Bräse, S.; Guenther, J.; Blümel, J.; Krishna, R.; Li, Z.; Zhou, H.-C. Porous Polymer Networks: Synthesis, Porosity, and Applications in Gas Storage/Separation. Chem. Mater. 2010, 22 (21), 5964-5972.

(11) Yuan, D.; Lu, W.; Zhao, D.; Zhou, H.-C. Highly Stable Porous Polymer Networks with Exceptionally High Gas-Uptake Capacities. Adv. Mater. 2011, 23 (32), 3723-3725.

(12) Makal, T. A.; Li, J.-R.; Lu, W.; Zhou, H.-C. Methane Storage in Advanced Porous Materials. Chem. Soc. Rev. 2012, 41 (23), 77617779.

(13) Trewin, A.; Cooper, A. I. Porous Organic Polymers: Distinction from Disorder? Angew. Chem., Int. Ed. 2010, 49 (9), 1533-1535.

(14) Batten, S. R. Topology of Interpenetration. CrystEngComm 2001, 3 (18), 67-72.

(15) Chen, B.; Eddaoudi, M.; Hyde, S. T.; O’Keeffe, M.; Yaghi, O. M. Interwoven Metal-Organic Framework on a Periodic Minimal Surface with Extra-Large Pores. Science 2001, 291 (5506), 1021-1023.

(16) Shekhah, O.; Wang, H.; Paradinas, M.; Ocal, C.; Schupbach, B.; Terfort, A.; Zacher, D.; Fischer, R. A.; Woll, C. Controlling Interpenetration in Metal-Organic Frameworks by Liquid-Phase Epitaxy. Nat. Mater. 2009, 8 (6), 481-484.

(17) Zhang, J.; Wojtas, L.; Larsen, R. W.; Eddaoudi, M.; Zaworotko, M. J. Temperature and Concentration Control over Interpenetration in a Metal-Organic Material. J. Am. Chem. Soc. 2009, 131 (47), 17040-17041.

(18) Yang, S.; Lin, X.; Lewis, W.; Suyetin, M.; Bichoutskaia, E.; Parker, J. E.; Tang, C. C.; Allan, D. R.; Rizkallah, P. J.; Hubberstey, P.; Champness, N. R.; Thomas, K. M.; Blake, A. J.; Schröder, M. A Partially Interpenetrated Metal-Organic Framework for Selective 
Hysteretic Sorption of Carbon Dioxide. Nat. Mater. 2012, 11 (8), $710-716$.

(19) O'Keeffe, M.; Yaghi, O. M. Deconstructing the Crystal Structures of Metal-Organic Frameworks and Related Materials into Their Underlying Nets. Chem. Rev. 2012, 112 (2), 675-702.

(20) O'Keeffe, M.; Peskov, M. A.; Ramsden, S. J.; Yaghi, O. M. The Reticular Chemistry Structure Resource (RCSR) Database of, and Symbols for, Crystal Nets. Acc. Chem. Res. 2008, 41 (12), 1782-1789.

(21) Ockwig, N. W.; Delgado-Friedrichs, O.; O’Keeffe, M.; Yaghi, O. M. Reticular Chemistry: Occurrence and Taxonomy of Nets and Grammar for the Design of Frameworks. Acc. Chem. Res. 2005, 38 (3), $176-182$.

(22) Alexandrov, E. V.; Blatov, V. A.; Kochetkov, A. V.; Proserpio, D. M. Underlying Nets in Three-Periodic Coordination Polymers: Topology, Taxonomy and Prediction from a Computer-Aided Analysis of the Cambridge Structural Database. CrystEngComm 2011, 13 (12), 3947-3958.

(23) Uribe-Romo, F. J.; Hunt, J. R.; Furukawa, H.; Klöck, C.; O'Keeffe, M.; Yaghi, O. M. A Crystalline Imine-Linked 3-D Porous Covalent Organic Framework. J. Am. Chem. Soc. 2009, 131 (13), $4570-4571$.

(24) Stewart, J. J. P. Optimization of Parameters for Semiempirical Methods V: Modification of NDDO Approximations and Application to 70 Elements. J. Mol. Model. 2007, 13 (12), 1173-1213.

(25) Korth, M.; Pitoňák, M.; Řezáč, J.; Hobza, P. A Transferable HBonding Correction for Semiempirical Quantum-Chemical Methods. J. Chem. Theory Comput. 2010, 6 (1), 344-352.

(26) Willems, T. F.; Rycroft, C. H.; Kazi, M.; Meza, J. C.; Haranczyk, M. Algorithms and Tools for High-Throughput Geometry-Based Analysis of Crystalline Porous Materials. Microporous Mesoporous Mater. 2012, 149 (1), 134-141.

(27) Reineke, T. M.; Eddaoudi, M.; Moler, D.; O’Keeffe, M.; Yaghi, O. M. Large Free Volume in Maximally Interpenetrating Networks: The Role of Secondary Building Units Exemplified by $\mathrm{Tb}_{2}(\mathrm{ADB})_{3}\left[\left(\mathrm{CH}_{3}\right)_{2} \mathrm{SO}\right]_{4} \cdot 16\left[\left(\mathrm{CH}_{3}\right)_{2} \mathrm{SO}\right]^{1}$. J. Am. Chem. Soc. 2000, 122 (19), 4843-4844.

(28) Choi, S. B.; Furukawa, H.; Nam, H. J.; Jung, D.-Y.; John, Y. H.; Walton, A.; Book, D.; O’Keeffe, M.; Yaghi, O. M.; Kim, J. Reversible Interpenetration in a Metal-Organic Framework Triggered by Ligand Removal and Addition. Angew. Chem., Int. Ed. 2012, 51 (35), 87918795. 